\title{
Modelo Praxeológico Extendido una Herramienta para Analizar las Matemáticas en la Práctica: el caso de la vivienda Maya y levantamiento y trazo topográfico
}

\author{
The Extended Praxeological Model: a tool to analyze mathematics in \\ practice - the case of Mayan house, survey and topographical drawing
}

\author{
Olda Nadinne Covián Chavez* \\ Avenilde Romo Vázquez ${ }^{* *}$
}

\begin{abstract}
Resumen
En esta comunicación se presenta el análisis de actividades relacionadas con la construcción, vivienda maya, levantamiento y trazo topográfico en la cultura egipcia. Debido a la naturaleza de estas actividades, los conocimientos matemáticos en uso hacen intervenir dos tipos de validaciones, teóricas y prácticas. Para analizar el rol de estos dos tipos de validaciones se utilizó un modelo praxeológico extendidoen el cual las validaciones prácticas tienen un rol fundamental.Su caracterización sienta la base para el desarrollo de diseños didácticos en la formación profesional de futuros técnicos en construcción.
\end{abstract}

Palabras-clave: Actividades de Construcción. Análisis Praxeológico. Formación Profesional. Conocimientos Matemáticos en Uso.

\begin{abstract}
In this paper we present analysis of building activities, Mayan house, survey and topographical drawing in the Egyptian culture. Due to the nature of these activities, the mathematical knowledge used brings into play two types of theoretical and practical validations. To analyze the role of these two types of validations we used an extended praxeologigal model. The practical validations have an important role, and in our analysis we have characterized these validations. This provides have an important basis to design didactical activities for training future construction technicians.
\end{abstract}

Keywords: Building Activities. Praxeological Analysis. Professional Training. Mathematics in Use.

\section{Introducción}

Las formaciones profesional-técnico ocupan, cada vez más, un lugar importante en la oferta de educación media superior. En México, en el ciclo escolar 2010-2011 ocuparon el 9\% de la matrícula total de alumnos, siendo el Colegio Nacional de Educación Profesional

\footnotetext{
* Ph.D. en Matemática Educativa del Centro de Investigación y de Estudios Avanzados del Instituto Politécnico Nacional (CINVESTAV-IPN). Dirección postal: Avenida Zacatenco 2508, Col. San Pedro Zacatenco, Del. Gustavo A. Madero, C.P. 07000, México D.F., México.E-mail: nadinne.olda@gmail.com.

${ }^{* *}$ Ph.D. en Didáctica de las Disciplinas en la Universidad Paris Diderot - Paris 7. Profesora Investigadora en el Programa de Matemática Educativa del Centro de Investigación en Ciencia y Tecnología Avanzada del Instituto Politécnico Nacional (CICATA-IPN). Dirección postal: Legaria 694, Col. Irrigación, Del. Miguel Hidalgo, C.P.
} 11500, México D.F., México.E-mail: avenilderv@yahoo.com.mx. 
Técnica (CONALEP) el que congrega el 70\% de los futuros profesionales-técnicos. A pesar del incremento de estas formaciones, son pocas las investigaciones desarrolladas para reconocer las necesidades matemáticas en los lugares de trabajo donde laboran sus egresados; lo que permitiría, en efecto, reconocer la pertinencia de los modelos de formación. Las investigaciones sobre el tema de las matemáticas del trabajo se han realizado hasta ahora, como lo señala Sträber (2005), en los países industrializados, Alemania, Australia, Estados Unidos, Francia, Inglaterra, y los Países Bajos. Algunos de estos estudios (VERGNAUD, 1996; NOSS; HOYLES; POZZI, 2000; KENT; NOSS, 2002; STRÄBER, 2005; ROMOVÁZQUEZ, 2009) han puesto de manifiesto que las matemáticas en el trabajo y/o en las prácticas profesionales, suelen ser invisibles, transpuestas, recompuestas o estar imbricadas con otros tipos de conocimientos.

Consideramos que estos resultados son importantes y pueden ser ampliados al estudiar otras actividades prácticas, como las de construcción. Esta elección se basa en que, en México, los profesionales-técnicos de la construcción reciben actualmente una formación matemática, y nos preguntamos cómo esa formación podría adoptar elementos de la práctica y relacionarlos con los contenidos matemáticos escolares. Es decir, antiguamente los constructores no recibían una formación matemática escolarizada, pero su práctica se desarrolló y mostró ser eficaz: durabilidad y estabilidad de las viviendas, adaptación de los diseños a regiones y climas, entre otras. Esto nos motivó a analizar actividades prácticas de construcción con dos objetivos: conocer qué matemáticas se usan y cómo las validaciones prácticas se relacionan con las validaciones matemáticas. Para lo cual, retomamos elementos del estudio de una práctica de construcción de vivienda maya reportados en Covián (2005) y los analizamos desde un enfoque antropológico-didáctico.

Asimismo, analizamos las actividades de levantamiento y trazo topográfico desarrolladas en la cultura egipcia, que aunque han pasado por procesos de institucionalización que las han vuelto más estables, siguen siendo parte de la topografía. Algunas de las preguntas que orientaron el análisis fueron: ¿cuáles son las matemáticas presentes en la construcción de la vivienda maya, en el levantamiento y trazo topográfico?; ¿en qué medida el reconocimiento de estas matemáticas puede ser útil para las formaciones técnicas de futuros profesionales de la construcción? Abordar estas preguntas desde un enfoque antropológico-didáctico supone un análisis de tareas, de técnicas empleadas y, sobre todo, de los elementos que permiten validarlas, justificarlas y explicarlas. La herramienta que nos pareció óptima para realizar dicho análisis es el modelo praxeológico extendido (CASTELA; ROMO-VÁZQUEZ, 2011). 


\section{Modelo praxeológico extendido: herramienta de análisis}

El modelo praxeológico extendido (CASTELA; ROMO-VÁZQUEZ, 2011) se inscribe en la Teoría Antropológica de lo Didáctico, la cual ofrece un modelo epistemológico para el estudio de la actividad humana. En Chevallard (1999) se reconoce a la praxeología $[T, \tau, \theta$, $\Theta$ ], como una unidad mínima de análisis de la actividad humana, sus cuatro componentes son: tipo de tarea $T$, técnica $\tau$, tecnología $\theta$ y teoría $\Theta$. La tarea es lo que se hace, la técnica es la manera en que se hace, la tecnología es un discurso que produce, justifica y explica la técnica; la teoría, a su vez, produce, justifica y explica la tecnología. El modelo praxeológico extendido considera, a diferencia del clásico, que la tecnología tiene dos componentes, teórica $\theta^{\text {th }}$ y práctica $\theta^{\mathrm{p}}$. Particularmente, la componente práctica es un discurso con seis funciones que permiten, describir, validar, explicar, facilitar, motivar y evaluar el uso de técnicas matemáticas en referencia a instituciones usuarias, no necesariamente matemáticas. El modelo puede expresarse de la siguiente manera:

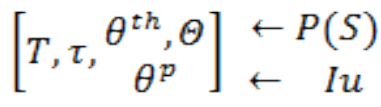

Donde $P(S)$ designa la institución productora de saberes e $I u$ la institución usuaria de dichos saberes. La práctica es entendida en este modelo como una institución que produce o traspone un conjunto de praxeologías que permiten resolver de manera eficaz y estable las tareas propias de dicha institución.

Las praxeologías que produce la práctica las denominamos como praxeologías prácticas en el sentido de que se producen, validan y justifican en referencia a la funcionalidad práctica. Por tanto, la práctica de construcción de la vivienda maya ocupa la posición de $I u$ puesto que las tecnologías que producen, explican y justifican las técnicas empleadas son validadas y legitimadas por su eficacia en la construcción misma. Es decir, las tecnologías no son evaluadas ni legitimadas dentro de una teoría, no se pretende demostrar su estabilidad a partir de lo abstracto, sino de su funcionalidad práctica. Por otra parte, las Matemáticas $P(M)$ y la Topografía $P(T)$ son instituciones del tipo $P(S)$, pero también pueden ocupar en el modelo la posición de $I u$ dependiendo de la tarea que se resuelva, de las técnicas en juego y, sobre todo, del bloque tecnológico-teórico $\left[\theta^{\text {th }}, \Theta\right]$ o tecnológico-práctico $\left[\theta^{\mathrm{p}}\right]$ que sustente dichas técnicas. Dicho de otra manera, las instituciones serán productoras cuando la componente tecnológica - validaciones, explicaciones, justificaciones - provengan de una teoría, y serán usuarias cuando la componente tecnológica provenga de la práctica. 


\section{En la investigación de Romo-Vázquez (2009) se utilizó el modelo praxeológico}

extendido para analizar actividades prácticas, proyectos de ingeniería, en los que aparecían

tareas no matemáticas realizadas con técnicas matemáticas y validadas en gran parte por

tecnologías prácticas. Dichas tecnologías fueron evidenciadas a partir de las funciones que

emergen de la componente práctica de la tecnología, las cuales se precisaron en Castela;

Romo-Vázquez, (2011) de la siguiente manera:

1.- Describir el tipo de tareas y la técnica. La producción de un discurso que caracteriza el tipo de tarea y los pasos que componen una técnica se considera como una pieza de saber que no es identificable por sí misma. Las acciones y el contexto social donde se sitúa la noción praxeológica en un sistema compartido se pueden identificar en la elaboración de un sistema de representaciones verbales y simbólicas. La producción de estos lenguajes y la descripción que ellos permiten hacer constituye una componente esencial del proceso de transmisión de una invención técnica.

2.- Validar la técnica. Corresponde a lo que generalmente se entiende como justificar. Los saberes considerados establecen que la técnica produce bien lo que ella dice que produce, que los pasos que la componen permiten alcanzar los objetivos que le son asignados. En el caso de las matemáticas, esta función es generalmente asegurada por los saberes, justificados por las teorías matemáticas. Sin embargo en otros contextos, los saberes validados experimentalmente en laboratorio o empíricamente en el uso pueden validar una técnica.

3.- Explicar la técnica. Se trata de saberes que permiten analizar cómo la técnica y sus diferentes pasos permiten conseguir los objetivos pretendidos. Contribuyen a la comprensión de las causas de los sujetos y están relacionadas a su cultura compartida.

4.- Facilitar la aplicación de la técnica. Los saberes considerados en esta función permiten a los usuarios utilizar con eficacia pero también con un cierto confort la técnica. Éstos son portadores de mejoras pero también de advertencias que permiten evitar errores y torpezas conocidas como frecuentes. Este dominio de saberes es el terreno privilegiado de las elaboraciones tecnológicas de los usuarios. Dicho dominio produce efectos retomados de descripciones que lo especifican al adaptarlo a las condiciones particulares del contexto institucional de utilización y el enriquecimiento de la memoria de las experiencias acumuladas.

5.- Motivar la técnica y los pasos que la componen. Estos saberes están orientados hacia la práctica. Ellos participan de una inteligencia de los fines: son los objetivos esperados que justifican racionalmente los pasos mostrando su razón de ser. Se trata de escribir una historia de la técnica que sitúe sus componentes, unas en relación con las otras: por qué (¿para hacer qué?) ¿se realiza tal paso en tal momento? Los saberes de motivación están seguidos por saberes sobre el tipo de tareas puesto que ellos analizan los objetivos. Permiten anticipar las etapas esperadas y entonces juegan un rol heurístico importante luego que la aplicación de la técnica necesita adaptaciones.

6.- Evaluar la técnica. Los saberes considerados en este rubro tienen que ver con el dominio, las condiciones y los límites de una técnica relativamente a las tareas del tipo T. Ellos pueden igualmente considerarse con el funcionamiento de la técnica desde el punto de vista de sus usuarios. La funciones evaluar, facilitar y motivar pueden estar bastante relacionadas: la puesta en evidencia de ciertas dificultades (evaluar) puede provocar al cabo de cierto tiempo la producción de mejoramientos (facilitar), la motivación es dada por la evaluación. (CASTELA; ROMOVÁZQUEZ, 2011, p. 88-89 ${ }^{1}$ ).

${ }^{1}$ Los nombres de las funciones no aparecen en foco en la publicación original. 
Estas seis funciones permiten analizar actividades prácticas, en particular reconocer el rol de las validaciones experimentales y cómo éstas se complementan o no con validaciones teóricas. Lo anterior, nos parece necesario para reconocer las necesidades matemáticas que aparecen en contextos extra-matemáticos, la manera en que se satisfacen y, más precisamente, cómo se legitiman y validan. Con el objetivo de analizar el rol de las validaciones experimentales y prácticas, consideramos para nuestro análisis actividades suficientemente estables. En particular, analizamos actividades de la práctica de construcción de la vivienda maya, expuesta en Covián (2005), y actividades de levantamiento y trazo topográfico en sus orígenes, particularmente en la cultura egipcia, para conocer qué técnicas matemáticas fueron generadas para desarrollarlas y, más aún, qué tecnologías las sustentaron.

El análisis se centra por tanto en tareas, destacando cómo su naturaleza motivó la construcción de técnicas matemáticas y de validaciones prácticas que poco a poco se fueron teorizando para resolver otros tipos de tareas más sofisticadas. La construcción, las matemáticas y la topografía son consideradas, en esta investigación, como instituciones de referencia, que nos permiten dar cuenta de las técnicas que eran utilizadas y cómo las reconoceríamos actualmente a partir de dichas instituciones.

\section{Análisis praxeológico de una práctica de construcción de la vivienda maya}

El estudio sobre la construcción de la vivienda maya (COVIÁN, 2005), tuvo como objetivo conocer y caracterizar el conocimiento matemático involucrado en dicha práctica. Se considera necesario precisar que esta práctica de construcción es muy antigua, según Thompson (2003) se tienen reportes desde el 1000 d.C. y permanece hasta nuestros días en comunidades de la península de Yucatán. La permanencia de esta práctica resulta sorprendente, por lo que, una de las preguntas que surgen es ¿qué tipo de matemáticas surgen en dicha práctica? Esta pregunta emerge al considerar que la práctica estudiada tiene lugar en una de las regiones menos occidentalizadas de México, los constructores, en su mayoría, no cuentan con antecedentes escolares y el oficio de constructor se transmite de generación en generación.

En Covián (2005) se analizaron diferentes documentos (DÍAZ, s.f.; PÉREZ, 1993) enfocados en el estudio de la vivienda tradicional maya y fueron producidos en la Facultad de Arquitectura de la Universidad Autónoma de Yucatán. Uno de éstos resultó muy importante, pues era un manual de construcción de la vivienda tradicional, el cual fue elaborado a partir de un estudio de campo. En dicho manual se solicitó a varios constructores explicitar las 
técnicas empleadas y se reportó una técnica general óptima para favorecer su difusión. Al igual que en este manual, en el trabajo de Covián (2005) se entrevistó a varios constructores tradicionales que relataron, a solicitud de la investigadora, las técnicas para construir una vivienda maya. En este escrito se presenta sólo el caso de uno de estos constructores, Gilberto, debido a que fue él quien narró con mayor detalle los elementos que componen la técnica, así como las razones de su funcionamiento. Estos elementos nos permitieron efectuar el análisis praxeológico de la práctica de construcción de la vivienda tradicional maya.

\subsection{Construcción de la vivienda tradicional maya}

La actividad principal que puede identificarse, para ser consistente con el análisis praxeológico, es la de Construcción de la Vivienda Tradicional Maya. En esta actividad se reconocieron dos tareas principales: trazar el plano de la vivienda y determinar la altura del techo.

\section{Tarea 1. Trazar el plano de la vivienda}

La primera tarea consiste en producir un plano de la vivienda (ver Figura 1). La Figura 1se realizó siguiendo la narración que efectúa Gilberto de cómo se hace el plano, reportada en Covián (2005, p.112-117). En dicha figura se observa la forma que adquiere la vivienda.

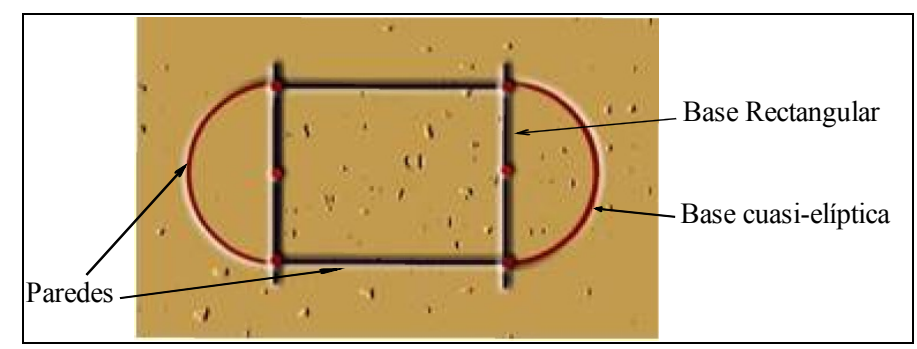

Figura 1 - Plano de la vivienda vista desde una toma aérea Fuente: desarrollado por los autores

Para trazar el plano anterior, reconstruimos, con base en la narración de Gilberto, la técnica que presentamos a continuación y que está compuesta de cuatro pasos:

1) Primero se determina la unidad de medida conocida como vara.Según Gilberto, una vara corresponde a cuatro cuartas y cinco cuartas es un metro (COVIÁN, 2005, p. 93).

2) Determinar el largo de la hamaca, señala Gilberto, permite conocer las dimensiones de la base rectangular de la vivienda. Gilberto afirma que lo máximo que puede medir una hamaca es $4 \mathrm{~m}$ o 4 varas. Hacemos notar que Gilberto utiliza indistintamente vara y metro, considerándolos equivalentes. 
3) Gilberto considera, para explicitar su manera de construir la vivienda, un largo de $4 \mathrm{~m}$ (que puede variar) y un ancho siempre constante, equivalente a 3 metros.

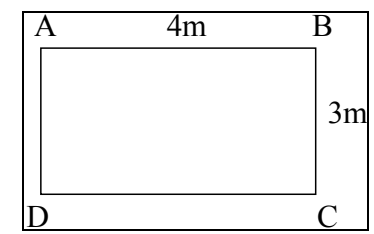

Figura 2 - Base rectangular de 4 × 3

Fuente: desarrollado por los autores

4) Luego, se trazan semicírculos teniendo como diámetro el ancho del rectángulo, es decir AD y BC (Figura 3).

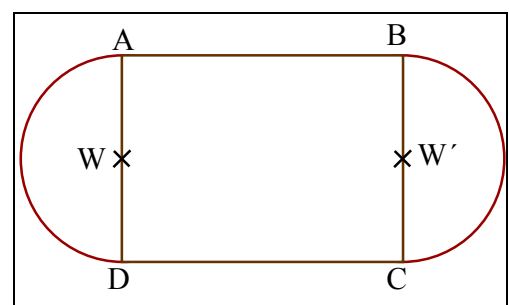

Figura 3 - Plano de la vivienda Fuente: desarrollado por los autores

Seguido, se sitúan cuatro postes en cada uno de los vértices, A, B, C y D de la base rectangular (Figura 2).

5) Cada poste se entierra medio metro y se dejan dos metros de longitud sobre el nivel del suelo.

Los primeros cuatro pasos de la técnica permiten determinar la forma geométrica de las paredes y la delimitación de la vivienda.Analicemos las validaciones tecnológicas que surgen. Primero se reconoce la necesidad de determinar una unidad de medida para establecer las dimensiones de la vivienda, las cuales dependerán del tamaño de la mano del constructor. Pues, recordemos que un metro es equivalente a cuatro o cinco cuartas. Dicha proporción de la vivienda, con respecto a la medida del constructor, se confirma en parte en el manual de construcción de la vivienda (DÍAZ, s.f. apud COVIÁN, 2005).

En dicho manual se menciona, explícitamente, que una vara corresponde a la medida que va del ombligo a los pies del constructor. Esto nos lleva a plantear que la motivación de la técnica está asociada a la proporcionalidad de la vivienda con respecto a las personas que la habitan, lo cual, se puede observar en el segundo y tercer paso de la técnica narrada por Gilberto. En éstos, también se observa la necesidad de dimensionar la longitud del rectángulo (Rectángulo $\mathrm{ABCD}$, ver Figuras 2 y 3) de acuerdo a la longitud de la hamaca. Un planteamiento que surge, y que en parte justifica matemáticamente la relación de la vivienda con respecto a la dimensión de la hamaca, puede ser la siguiente: $x=$ la medida de la persona 
que descansará en la hamaca; $f(x)=$ es la función que determina la longitud ${ }^{2}$ de la hamaca de la persona; $g(f(x))$ = es la función que determina el largo de la estructura (COVIAN, 2005, p. 164-165). Lo cual corresponde a reconocer un modelo matemático que subyace los pasos 2 y 3 de la técnica reconstruida.s

En el trabajo de Covián (2005) no se precisa el rango de variabilidad del largo de las bases rectangulares, sino que el máximo puede ser $4 \mathrm{~m}^{3}$.Esta técnica de construcción se basa en un plano geométrico que requiere simetría, visión espacial, y proporcionalidad. La simetría axial se refleja en el plano de la vivienda, pues sí se traza un eje imaginario que pase por el punto medio de $\mathrm{AB}$ y por el centro del rectángulo, se observa que la parte derecha del plano es una reflexión de la parte izquierda.De la misma manera, si se traza un eje que pase por WW' la sección de arriba se reflejará sobre la de abajo.

Analicemos de igual forma la técnica de construcción del plano de la vivienda del manual de construcción (DÍAZ, s.f. apud COVIÁN, 2005):

Se trazan dos circunferencias tangentes con radio igual a 3 varas, donde la vara es igual a la mitad de la altura de la persona que habitará la casa.

Después de trazar las circunferencias tangentes, se procede a obtener el trazo de la mediatriz de ambos círculos y ahí se localizarán los horcomes ${ }^{4}$ o columnas de la casa (COVIÁN, 2005, p. 166).

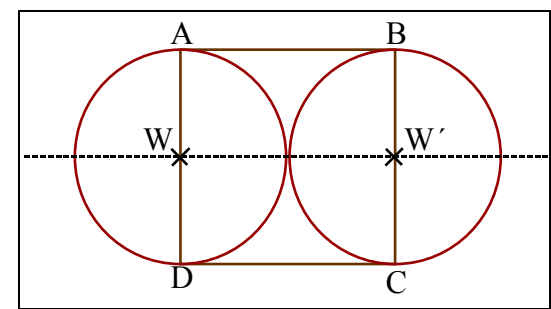

Figura 4 -Trazo del plano de la casa que presenta el manual Fuente: desarrollado por los autores

La técnica descrita en el manual muestra que la distancia de W a W', (de Horcom a Horcom) es de 6 varas. En este caso, se observa que a diferencia del plano de Gilberto el rectángulo $\mathrm{ABCD}$ (Figura 3) se torna en un cuadrado ABCD (Figura 4). Esto permite observar que para la técnica presentada en el manual la importancia no radica en la longitud de la hamaca para determinar la distancia de AB y DC (Figura 3). Sin embargo, sí conservan la necesidad de construir con base en la proporción del cuerpo, puesto que, la unidad de medida (la vara) tiene como principal equivalencia la medida del cuerpo de la persona que

\footnotetext{
${ }^{2}$ En el texto original aparece valor, pero consideramos que la palabra longitud es más precisa.

${ }^{3}$ La variabilidad del largo se constató en la visita a la comunidad Muna, donde las casas a pesar de conservar la misma estructura tienen dimensiones distintas.

${ }^{4}$ Los horcomes son los postes, que deberán ponerse en los vértices de la base. Los puntos señalados como A, B, C y D en la Figura 4. 
construye (longitud del ombligo a la planta del pie). Por lo que, la variación se establecerá de acuerdo a la medida que tenga la persona que construirá la vivienda o habitará en ella. Lo anterior debido a que, la persona que construye es generalmente el jefe de familia o quien la habitará.También se observa que la forma de la vivienda se conserva en ambas técnicas y lo único que varía es la técnica geométrica de construcción, sin embargo, las unidades de medida que motivan ambas técnicas es la medida de la persona que habitará la casa para garantizar la comodidad de ésta.

Por otra parte, el plano construido en 2D permite al constructor visualizar espacialmente la vivienda que está construyendo y darse cuenta de que el dimensionamiento realizado permite levantar una vivienda de base cuasi-elíptica; el constructor no detalla los elementos que validan tal dimensionamiento. Sin embargo, las validaciones prácticas permiten construir una vivienda adecuada para resistir tanto las altas temperaturas de la región como los fuertes vientos provocados por huracanes o tifones que azotan esta misma zona (PÉREZ, 1993).

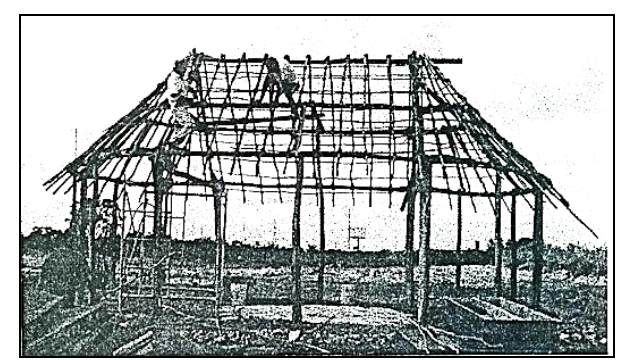

Figura 5 - Fotografía de la estructura de la vivienda Fuente: Diaz, s.f. apud Covián (2005, p. 146)

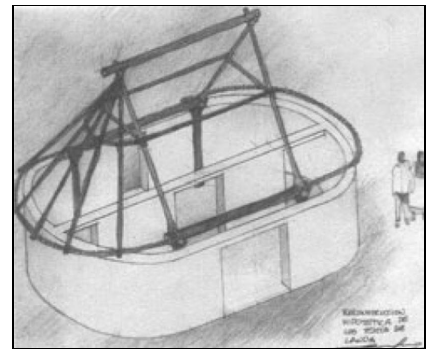

Figura 6 - Vivienda tradicional maya Fuente: Baños (2003, p. 179)

Como puede verse en las Figuras 5 y 6, el plano permitirá a los constructores asegurar una forma cuasi elíptica de las paredes, proyectando la misma forma de la base en la parte superior. La motivación de la forma geométrica de la estructura parece venir de la necesidad de generar una vivienda estable, que resista los fuertes vientos que azotan la región. Para lograr tal geometría, un elemento importante es la ubicación simétrica de postes sobre la base, seis postes sobre cada semicírculo, lo que parece asegurar la proyección del plano en la parte superior y la estabilidad de la vivienda.Asimismo, esto se logra gracias al uso de materiales perecederos, paja y madera que pueden adaptarse fácilmente a cualquier tipo de estructura o forma geométrica.

\section{Tarea 2. Determinar la altura del techo}

1) Para determinar la altura del techo, Gilberto ubica los puntos medios de los segmentos $\mathrm{AD}$ y $\mathrm{BC}, \mathrm{W}$ y W' respectivamente (ver Figura 7). 


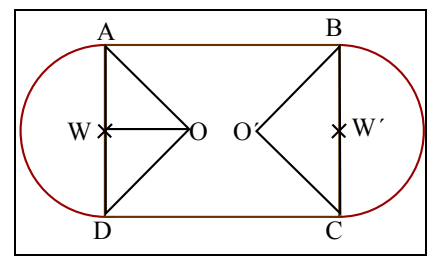

Figura 7 - Plano de la vivienda en el que se incluye la altura del techo Fuente: desarrollado por los autores

Se observa que las perpendiculares sobre cada punto medio (WO y W'O') miden 2.5 metros, respectivamente.

2) Se levantan los triángulos AOD y BO'C hasta obtener la estructura de la vivienda como se muestra en la Figura 8.

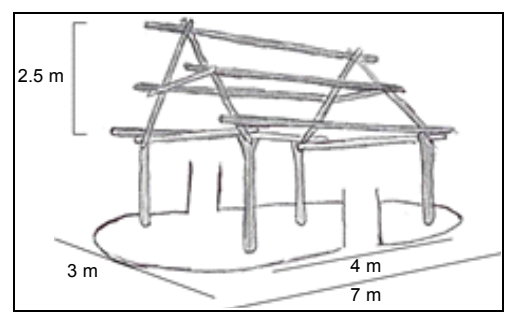

Figura 8 - Estructura de la vivienda

Fuente: desarrollado por los autores

Volviendo a la descripción que hace Gilberto de su técnica y, particularmente, en lo que tiene que ver con la construcción del techo, aparece una noción que nos llama mucho la atención y que en el trabajo de Covián (2005) ha sido llamada inclinación. Para evidenciarla, presentamos, a continuación, cómo Gilberto describe dicha técnica. En Covián (2005, p. 8788) aparece $^{5}$ :

1. G: Si quieres ponerle bajo así, para que no acumule el agua (señalando la altura de la casa respecto a la inclinación como se muestra en la Figura 9).

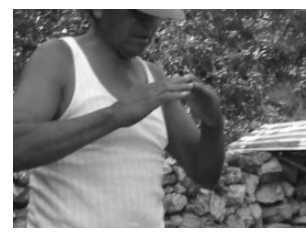

Figura 9-Gilberto mostrando la inclinación del techo (COVIÁN, 2005, p. 87)

2. G: Porque si lo pones muy así, inclinado pues legalmente cuando venga el agua, penetra (Mostrando la inclinación del techo de la casa con una abertura mayor, como se muestra en la Figura 10).

\footnotetext{
${ }^{5}$ El nombre y número de la figura se han modificado para esta comunicación y se tomaron de Covián (2005, p. 174-175). 


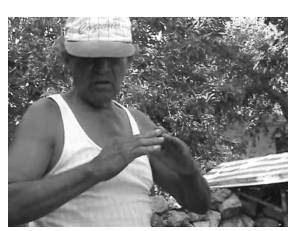

Figura 10 - Gilberto explicando que a más inclinación del techo el agua entra a la casa

Fuente: Covián $(2005$, p. 87)

3. G: Cuando está así, cuando caiga el agua, abajo (Mostrando la inclinación del techo de la casa con una pendiente más pronunciada, resultando una abertura más pequeña Figura 11).

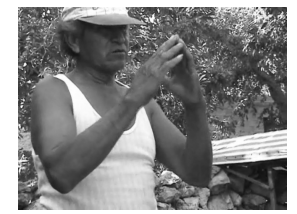

Figura 11 - Gilberto mostrando la altura que debe tener el techo para que el agua no entre

Fuente: Covián $(2005$, p. 88)

4. G: Ahora por ejemplo si quieres ponerle lámina, pues tienes que poner un declive así porque si es de lámina resbala (la inclinación del techo de la casa es menos pronunciada, Figura 12).

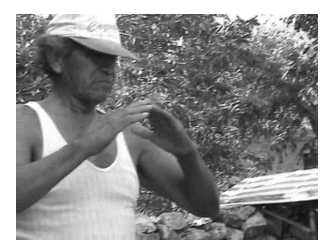

Figura 12 - Gilberto mostrando la inclinación del techo cuando el material utilizado cambia

Fuente: Covián (2005, p. 88)

5. G: Pero si es para una casa así (señalando la casa de materiales perecederos con un techo de paja), entonces tienes que ponerle altura para cuando venga el agua, abajo, así es.

6. E: ah! ¿entonces depende de la caída del agua no?

7. G: Sí, si tiene más altura, más mejor.

La explicación hecha por Gilberto, reportada previamente, muestra las razones que fundamentan la elección tanto de la inclinación como de la altura del techo. En su discurso, Gilberto señala cómo elegir una inclinación para evitar la entrada de la lluvia en la casa, incluso muestra con sus manos tres diferentes inclinaciones, señalando la correcta y explicitando que ésta también se decide en relación a los materiales que serán usados. Lo que vemos aquí es que la forma de la vivienda se piensa, se decide y se construye en relación al clima y a los materiales usados.

Analicemos la misma descripción de la técnica hecha por Gilberto, no sin olvidar que el ancho de la casa, según las medidas que nos proporciona Gilberto, es igual a $3 \mathrm{~m}$ y la altura del techo es de $2.5 \mathrm{~m}$. Por tanto tenemos que la Ticera (ver Figura 13) forma un triángulo cuya base es igual a $3 \mathrm{~m}$ y cuya altura es igual a $2.5 \mathrm{~m}$ como se ilustra en la Figura 13. 


\section{Figura 13 - Triángulo que forma la Tijera o Ticera del techo Fuente: Covian (2005, p. 176)}

Calculemos el ángulo de inclinación $\alpha$ que tiene el segmento $l$, el cual representa el lado de un triángulo que forma la Ticera. Al resolver $\operatorname{Tan} \alpha=\frac{2.5}{1.5}$. De lo que se obtiene que $\alpha=59^{\circ} 2^{\prime}$. Podemos decir, a partir del discurso de Gilberto, que él establece un parámetro de variación, donde lo que varía es la altura del triángulo y la base y el ángulo - formado por la base y uno de los lados ángulo - son constantes. En la justificación hecha por Gilberto basta centrarse en la altura del triángulo y no en el ángulo, éste variará en razón de la variación de la altura y así controlará la inclinación del techo.

La medida que se obtiene en la técnica narrada por Gilberto es una aproximación bastante cercana a la medida que Pérez (1993 p. 38) considera la óptima para la construcción de este tipo de viviendas resistente al embate del clima. Asimismo, esta inclinación asegurará un clima adecuado al interior, lo que permitirá enfrentar las altas temperaturas de la región. Por último, se sigue con una serie de pasos que permiten finalizar la vivienda que tiene la estructura que se describió anteriormente. La Figura 14 muestra viviendas mayas de Muna, Yucatán, México. Nos parece importante mencionar que todos los materiales utilizados son perecederos y que, en promedio, estas viviendas resisten diez años sin mantenimiento, y más tiempo, dependiendo del mantenimiento dado.

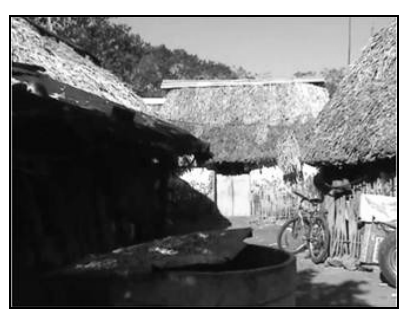

Figura 14 - Vivienda maya de Gilberto

Fuente: Covián (2005, p. 162)

Analizar la práctica de construcción de la vivienda maya a través de un enfoque antropológico-didáctico nos permite reconocer tareas en las que intervienen conocimientos matemáticos, construcción del plano de la base y determinación de la inclinación del techo. Estas dos tareas fueron identificadas y analizadas primeramente a partir del discurso producido por Gilberto y, posteriormente, cotejadas con un manual de construcción. Lo que 
nos permitió ver la proximidad de las técnicas y de las validaciones teóricas. En cuanto a las validaciones prácticas éstas aparecen de manera más evidente en el discurso de Gilberto: lo que motiva las dimensiones del plano es la estatura de la persona que habitará la casa; la inclinación del techo se determina en relación al clima y a la altura de la casa, esta última está también relacionada con el habitante de la casa. El ángulo de inclinación se determina en relación a la altura que debe tener la casa. Muy en particular esta justificación muestra que es la práctica la productora de validaciones, lo cual se confirma porque Gilberto no tiene antecedente escolar, el uso que hace de elementos matemáticos, figuras, propiedades, relaciones, no es sino a la luz de la construcción de la vivienda maya.

Analizaremos en la siguiente sección, dos actividades más relacionadas con la construcción, pero específicamente con el trabajo topográfico, el contexto elegido es la cultura egipcia, pues consideramos que al igual que el contexto maya, las validaciones prácticas juegan un rol fundamental.

\section{Análisis praxeológico del levantamiento y trazo topográfico en la cultura egipcia}

Las actividades de levantamiento y trazo topográfico forman parte indispensable de la topografía y por tanto de la formación de futuros Profesionales Técnicos en Construcción. Por ejemplo, en el CONALEP, institución formadora de dichos profesionales (bachillerato profesional técnico, 3 años) se imparte un curso de levantamiento, durante el segundo semestre, y un curso de trazo topográfico, durante el tercero. Es decir, estas actividades ocupan un lugar importante en el plan de estudios de dicha institución.

Para conocer las razones de esta importancia, analizamos el libro de referencia del curso de enseñanza de la topografía, en el cual se afirma “[...] el levantamiento es uno de los más viejos artes practicados por el hombre, porque desde épocas tempranas ha sido necesario marcar límites y dividir la tierra. Es una operación técnica que consiste en medir directamente el terreno" (GARCÍA, 2003, p. 4).

Esta afirmación nos motivó a conocer cómo surgen el levantamiento y trazo topográfico y, más específicamente, el rol de los conocimientos matemáticos en su evolución. Para lo cual, analizamos el desarrollo de la topografía en la cultura egipcia. Consideramos necesario señalar que las evidencias sobre dicho desarrollo son muy pocas, por lo que realizamos una reconstrucción praxeológica. Dentro de ésta, el interés principal fue caracterizar las praxeologías en juego, que en efecto evidenciarían el uso de técnicas y 
tecnologías matemáticas y topográficas que influyeron la consolidación de la topografía (como institución).

\subsection{Topografía y matemáticas, una relación muy antigua}

El levantamiento y el trazo topográfico son actividades muy antiguas; según algunos textos (GARCÍA, 2003) estas surgieron por la necesidad de medir y de delimitar terrenos. Bell (1985), Boyer (2007) y Struik (2008) reconocen que el origen de la trigonometría e incluso parte de la geometría, se encuentra en estas actividades, medición de terrenos, de la tierra misma y del levantamiento de planos.

Se sometió el número al servicio de la economía y del comercio; se aclaró la percepción de la forma con medidas empíricas, aplicándola a la astronomía, al levantamiento de planos y a la ingeniería [...] (BELL, 1985, p. 38).

[...] la trigonometría, originada en el levantamiento de planos, la astronomía y la geometría fueron absorbidas siglos más tarde en el análisis que había generalizado la geometría. (BELL, 1985, p. 30).

Nuestro estudio tiene por objetivo reconocer cómo en dichas actividades se motivó la producción y el uso de técnicas matemáticas (trigonométricas y geométricas). En específico, nos interesamos en reconocer el tipo de validaciones utilizadas y cómo éstas permitieron estabilizar ciertas técnicas matemáticas hasta nuestros días. Reconocer lo anterior, nos permitirá reflexionar sobre el origen de la relación entre la topografía y la matemática. Y más aún, tener elementos para sustentar, en futuras investigaciones, una propuesta didáctica para una formación de futuros profesionales técnicos en construcción o topógrafos.

\subsubsection{Del levantamiento y trazo topográfico a la geometría concreta}

Herodoto (484-425 a.C.), señala que las inundaciones anuales provocadas por el crecimiento del río Nilo, requerían la ubicación y reubicación de espacios de cultivo. Delimitar terrenos motivó, por tanto, el desarrollo de mediciones, cálculos y descripciones de superficies a través de triángulos, cuadriláteros y círculos, como se evidencia a continuación:

Sesotris... dividió las tierras de Egipto entre sus habitantes... Si el río se llevaba una parte de la porción asignada a un hombre... el rey enviaba a otras personas para examinar y determinar, por medio de una medición, la extensión exacta de la pérdida... A partir de esta práctica, creo yo, es como se llegó al conocimiento de la geometría en Egipto en primer lugar, de donde pasó más tarde a Grecia. (HERODOTO apud BOYER, 2007, p. 29) 
La anterior, parece referirse a lo que ahora se denomina como levantamiento y trazo topográficos ${ }^{6}$. En este período de florecimiento de la cultura egipcia, los agrimensores y constructores de pirámides eran los que producían las técnicas para llevar a cabo las tareas de delimitación de terrenos. Una de las técnicas utilizadas fue la medición, realizada principalmente con cuerdas de nudos (Figura 15). Las figuras predominantes fueron los triángulos rectángulos de catetos 3 y 4 e hipotenusa 5 , aunque como veremos posteriormente el uso de la triada 3, 4 y 5 no se asocia al conocimiento del teorema de Pitágoras, lo que permitía determinar perpendiculares. Lo anterior, se documenta como sucedido alrededor del 3000 a. C. (DE LA CRUZ; MESA; CUARTERO, 1998).

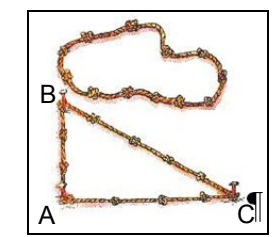

Figura 15 - Cuerdas utilizadas por los egipcios Fuente: García-Benedito (s.f.)

Las tareas solicitaban, esencialmente, efectuar mediciones cuyas técnicas implicaban cálculos con fórmulas, reportadas en el Papiro de Rhind, como se muestra en la siguiente cita:

El área del triángulo fue encontrada como la mitad del producto de la base por la altura; el área de un círculo con diámetro $d$ fue dado como $(d-d / 9)^{2}$, lo que condujo a un valor de $\pi$ de $256 / 81=3.1605$. Además existían fórmulas para volúmenes de sólidos, tales como el cubo, el paralelepípedo y el cilindro circular, todos concebidos concretamente como recipientes, principalmente de granos (STRUIK, 2008, p. 34).

Es interesante analizar como en esta cita Struik (2008) señala que estas fórmulas, que gozan de gran exactitud, fueron concebidas a partir de lo concreto, del uso de recipientes, principalmente de granos. Lo anterior nos hace suponer que la validación práctica sustenta la exactitud de la fórmula. Struik (2008) señala que el resultado más importante de la matemática egipcia es, sin duda, la fórmula para calcular el volumen del tronco de una pirámide cuadrada. La importancia de todos estos resultados se debe en parte a que los egipcios no conocían el teorema de Pitágoras, como lo señala Bell (1985):

Hasta el año 1923 se suponía que los egipcios conocían ese teorema por lo menos en el caso $5^{2}=4^{2}+3^{2}$ porque se decía que los agrimensores egipcios habían usado esta propiedad de los números 5, 4 y 3 para trazar ángulos rectos al orientar los edificios. Pero en la actualidad se afirma que, aunque es posible que los números puedan haberse usado con este fin, los egipcios no conocían ni un solo caso de la ecuación pitagórica $c^{2}=a^{2}+b^{2}$, porque no existe ninguna prueba documental de que la conocieran. Puesto que $c, a$, y $b$ son los lados del triángulo rectángulo solamente si

\footnotetext{
6 El levantamiento topográfico comprende dos actividades, ir al terreno y efectuar las mediciones correspondientes y tomar datos. Con los datos obtenidos se procede a efectuar cálculos y un plano o planta topográfica. El trazo topográfico comprende dos actividades, proyectar o diseñar elementos (edificaciones nuevas) en la planta topográfica, con ésta se va posteriormente al terreno, se efectúan medidas y trazos correspondientes para plantar estos nuevos elementos en el terreno.
} 
$c^{2}=a^{2}+b^{2}$ estamos en presencia de un enigma histórico interesante, esto es, cómo los egipcios adivinaron lo que necesitaban (BELL, 1985, p. 49).

Nuestra hipótesis es que los agrimensores egipcios comprobaron/validaron prácticamente la relación $5^{2}=4^{2}+3^{2}$, ya que medían las dimensiones de los terrenos, lo que les permitía constatar la relación anterior. Una vez más nos encontramos en el uso (incluso podría decirse en el origen) de las relaciones entre lo que hoy conocemos como catetos e hipotenusa y no en la importación de un teorema para resolver un problema práctico. Analizamos, a continuación, el levantamiento y trazo (delimitación de terrenos) los cuales son identificados como tipos de tareas, precisando tres tareas: medición de distancias entre los lados de un terreno, medición del terreno para trazo de figuras geométricas sobre el terreno y medición de la superficie del terreno para determinar el área.

\section{Tipo de Tarea 1. Delimitar terrenos (Levantamiento y Trazo Topográfico)}

Tarea 1 Delimitar terrenos cercanos al río Nilo.

Técnica 1 para delimitar el terreno se hacía una primera medición del posible contorno y se aproximaba a una figura geométrica conocida, un cuadrado, un rectángulo, un triángulo, un trapecio o un círculo. Luego se medían distancias entre los lados, utilizando una cuerda de nudos, existiendo la misma distancia entre dos nudos, para medir cada lado del terreno.

Tecnología $\theta^{\text {th }}$ Establecimiento de una unidad de medida que garantice la precisión de la medición. Reconocimiento de las propiedades de las figuras geométricas, cuadrado, rectángulo, trapecio, triángulo y círculo, así como de las rectas perpendiculares y del triángulo rectángulo. Uso de las fórmulas de área de las figuras geométricas antes mencionadas.

Tecnología $\theta^{p}$ Elección de una cuerda anudada para medir sobre el terreno los lados de una figura, la flexibilidad de la cuerda permitía trazar líneas lo más cercano a líneas rectas sobre un terreno que difícilmente era plano. El uso de esta cuerda parece estar motivado por las condiciones del terreno y por la búsqueda de precisión; una sofisticación del instrumento, sustentado en conocimientos geométricos - restringidos a las propiedades de las figuras conocidas.

Aunque la flexibilidad está asociada al instrumento de medida, reconocemos que existe una idea de línea recta que obliga a elegir este instrumento y a adaptarlo al tipo de tarea.

Además, podemos decir que una motivación para delimitar adecuadamente los terrenos era la de garantizar un reparto equitativo de tierras.

Tipo de Tarea 2. Delimitar terrenos (Trazo Topográfico). A diferencia de la tarea anterior, aquí se considera que existe un plano de los terrenos cercanos al Nilo y debido a la inundación 
la delimitación sobre los terrenos se pierde. Entonces, los agrimensores modifican el plano para reubicar los terrenos.

Tarea 2. Medición del terreno para trazo de figuras geométricas sobre éste. Esta tarea supone que el terreno que se desea acotar es plano o que no tenga tantos elementos (desniveles o lagos) que impidan efectuar las mediciones.

Técnica 2. Se elige un punto (A) sobre el terreno. Utilizando la cuerda de 12 nudos, se mide una distancia de 4 nudos hacia otro punto (C) suponiéndolo parte de la misma recta.

Se elige otro punto (B) sobre una recta perpendicular a AC con medida 3 nudos. Finalmente, se unen los puntos A y $\mathrm{C}$ con la cuerda de 5 nudos, obteniendo un triángulo rectángulo $\mathrm{ABC}$ (Figura 15).

Posteriormente, se mide desde el punto A y sobre la recta AB la distancia a medir. Lo cual, puede ser con la misma cuerda. Finalmente, se determina otro punto D y desde éste se vuelve a levantar otra perpendicular.

Esta técnica se sigue hasta trazar la figura deseada. (Figura $16^{7}$ )

Tecnología $\theta^{\text {th }}$ Propiedades de las rectas perpendiculares.Reconocimiento del caso particular del teorema de Pitágoras $\mathrm{a}^{2}+\mathrm{b}^{2}=\mathrm{c}^{2}$ donde $\mathrm{a}=3, \mathrm{~b}=4$ y $\mathrm{c}=5$, el cual asegura la perpendicularidad de las rectas AC y BC.

Reconocimiento de las propiedades geométricas de figuras conocidas (cuadrados, rectángulos, triángulos, trapecios) y así delimitar terrenos.

Tecnología $\theta^{p}$. Diferenciar tipos de terrenos, pues esta técnica sólo es aplicable a terrenos planos, los inclinados o con desniveles requieren de proyectar el plano a través de postes para poder utilizarla. Suponemos que el uso de los valores 3, 4 y 5 para asegurar la perpendicularidad de rectas se debe a su facilidad de aplicación. Elementos que permiten elegir el punto A sobre el terreno (visible, accesible). Elementos que permiten el trazo de perpendiculares sobre el terreno (evitar desniveles).Todos estos elementos permiten evaluar la elección de la técnica utilizada, pues se lograba hacer un dimensionamiento del terreno. 


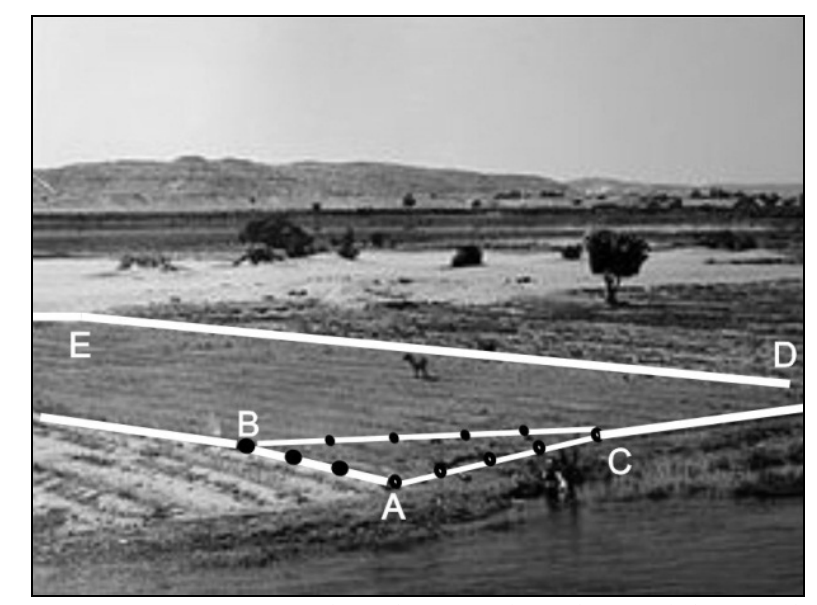

Figura 16 - Campos de cultivo en las riberas del río Nilo Fuente: Wikipedia, España.

Tipo de Tarea 3. Levantar el terreno. Delimitación del terreno.

Tarea 3. Medir la superficie del terreno para determinar el área.

Técnica 3. Uso de fórmulas para el cálculo de área de triángulos, cuadriláteros y círculos, los cuales se aproximarán en forma a los terrenos (reportados e institucionalizados por los escribas de la época, principalmente en el papiro de Rhind).

Tecnología $\theta^{\text {th }}$. Las fórmulas utilizadas permitían calcular el área del Triángulo, Rectángulo, Trapecio y Círculo. La cuales, están reportadas y desarrolladas en principalmente en el Papiro de Rhind.

Tecnología $\theta^{p}$. Posterior a las inundaciones que provocaba el Rio Nilo, las delimitaciones de las tierras se perdían. Esto motivaba a los encargados de la época, como agrimensores o geómetras midieran la superficie de los terrenos para calcular el área y así garantizar el reparto equitativo de tierras.

El análisis de estas tareas topográficas nos muestra la complejidad de acceder a las técnicas y tecnologías utilizadas por los egipcios, pues se cuenta con poca evidencia. Sin embargo, a partir del análisis de libros históricos se propuso una reconstrucción de técnicas y tecnologías lo que permitió analizar el tipo de praxeologías en juego en estas actividades y, más particularmente, el rol de las validaciones teóricas y prácticas. Al tener disponibles las fórmulas para el calcular el perímetro y área de varias figuras geométricas, los agrimensores aproximan los terrenos a estas formas geométricas, por lo que el trazo de perpendiculares se vuelve una técnica central. El uso de la triada 3, 4 y 5 validada, prácticamente permite ser el elemento tecnológico clave en el desarrollo de estas actividades. Consideramos que los tipos de tareas que emergen en la práctica, sus técnicas y tecnologías pueden ocupar un lugar en la formación de futuros técnicos en construcción, aportando una auténtica relación entre teoría y práctica. 


\section{Conclusión}

En este artículo hemos analizado diferentes actividades prácticas relacionadas a la construcción, con el objetivo de reconocer los conocimientos matemáticos en uso y, más precisamente, el rol de las validaciones prácticas asociadas a dichos conocimientos. En las tareas asociadas a la construcción de la vivienda maya hemos constatado cómo la motivación de las técnicas tiene que ver con la construcción de una vivienda adecuada para el habitante de la casa, resistente y apta al clima. Para lo cual, la inclinación del techo resulta fundamental, las explicaciones producidas por Gilberto muestran cómo la inclinación se decide tanto por una relación matemática entre la altura y uno de los ángulos agudos del triángulo rectángulo e isósceles, como por el tipo de material que se va a utilizar. Asimismo, podemos ver cómo la forma cuasi elíptica de la vivienda se proyecta a través de postes que van de la base de la vivienda a la base del techo. Los elementos que permiten decidir el número de postes (6 en el caso analizado) no son explicitados, pero todo parece indicar que es la estabilidad de la vivienda (validación práctica).

El análisis de las tareas topográficas muestra el rol fundamental de las validaciones prácticas que, históricamente, permitieron incluso validar las fórmulas de área y perímetro de figuras geométricas. El uso de la triada 3, 4 y 5 para el trazo de perpendiculares y el tratamiento de terrenos planos y terrenos con desniveles es sumamente interesante y nos parece ser una evidencia de cómo el tipo de tarea propicia un tipo de validación que surge en el contexto de uso. El modelo praxeológico extendido caracteriza funciones de las validaciones prácticas que permiten realizar análisis praxeológicos de tareas que emergen y se desarrollan en la práctica.

A partir de las tareas prácticas analizadas puede verse cómo las validaciones son tanto teóricas y prácticas. Lo anterior nos parece ser una base para el diseño de secuencias y/o dispositivos didácticos que puedan considerar el tratamiento didáctico de estos dos tipos de validaciones, particularmente para las formaciones tecnológicas y profesionales.

\section{Referencias}

BAÑOS, O. Hamaca y cambio social en Yucatán. Revista Mexicana del Caribe, México, D.F., v. 15, n. 8, p. 16-214, 2003. Disponible en: $<$ http://www.redalyc.org/articulo.oa?id=12881507>. Acceso en: 7 de mar. 2013.

BELL, E. T. Historia de las matemáticas. 2. ed. México, D.F.: Fondo de Cultura Económica, 1985. 
BOYER, C. Historia de la Matemática. México, D.F.: Alianza editorial, 2007.

CASTELA, C; ROMO-VAZQUEZ, A. Des mathématiques a l'automatique: étude des effets de transposition sur la transformée de Laplace dans la formation des ingénieurs. Recherches en Didactique des Mathématiques. Grenoble, v. 31, n. 1, p. 79-130. 2011.

CHEVALLARD, Y. L'analyse des pratiques enseignantes en théorie anthropologique du didactique. Recherches en didactique des mathématiques. Grenoble, v. 19, n. 2, p. 221-266. 1999.

COVIÁN, O. El papel del conocimiento matemático en la construcción de la vivienda tradicional: el caso de la cultura Maya. 2005. 192 f. Tesis (Maestría en Ciencias en Matemática Educativa) Centro de Investigación y de Estudios Avanzados, Instituto Politécnico Nacional, México, 2005.

DE LA CRUZ, J. L.; MESA, J. L.; CUARTERO, A. Evolución histórica de la instrumentación topográfica. Boletín del Instituto de Estudios Giennenses. Jaén, v. 169, p. 63-646. 1998.

DÍAZ, D. Manual de Auto construcción de la casa Maya. Investigación no publicada, Universidad Autónoma de Yucatán. Mérida Yucatán, México. s.f.

GARCÍA, F. Curso básico de topografía: planimetría, agrimensura, altimetría. México, D.F.: Editorial Pax México, 2003.

GARCÍA-BENEDITO, M. A. A. La matemática en el antiguo Egipto. Disponible en:

${ }^{<}$http://www.jimena.com/egipto/apartados/mates.htm\#geometria> Acceso en: 07 mar. 2013.

KENT, P.; NOSS, R. The mathematical components of engineering expertise : The relationship between doing and understanding mathematics. In: I.E.E. SECOND ANNUAL SYMPOSIUM ON ENGINEERING EDUCATION, $2^{\text {nd }}, 2002$, London. Proceedings of the IEE Second Annual Symposium on Engineering Education: Professional Engineering Scenarios 2. London U.K: Institution of Electrical Engineers, 2002. p. 39/1-39/7.

NOSS, R.; HOYLES, C.; POZZI, S. Working Knowledge: Mathematics in use. In: BESSOT, A.; RIDGWAY, J. (Ed.). Education for Mathematics in the workplace. Dordrecht: Kluwer Academic Publishers, 2000. p. 17-35.

PÉREZ, S. Transformación de la vivienda rural en Yucatán: Estudio de caso. Cuadernos de Arquitectura, Mérida, n. 6, p. 57-73, 1993.

ROMO-VAZQUEZ, A. La formation mathématique des futurs ingénieurs. 2009. $305 \mathrm{f}$. Thèse (Doctorat en didactique des mathématique) - IREM, Université Paris Diderot Paris 7, Paris, 2009.

STRUIK, D. Historia concisa de las Matemáticas. México, D.F.: Consejo Editorial del Instituto Politécnico Nacional, 2008.

STRÄBER, R. A propos de la transition du secondaire vers le monde du travail. In: ECOLE D'ETE DE DIDACTIQUE DES MATHEMATIQUES, 13., 2005, Ste Livrade. Perspectives en didactique des mathématiques. Actes de la XIIIème Ecole d'Eté de Didactique des Mathématiques. Fontenay-Le-sCompte: La pensé sauvage, 2005, p. 177-186.

THOMPSON, J. Grandeza y Decadencia de los Mayas. México, D.F.: Fondo de Cultura Económica, 2003. 
VERGNAUD, G. Au fond de l'action, la conceptualisation. In: BARBIER, J. M. (Ed.). Savoirs théoriques et savoirs d'action. Paris: Presses Universitaires de France, 1996. p. 275-292.

Submetido em Outubro de 2012. Aprovado em Fevereiro de 2013. 